\title{
Na interface entre Lógica Formal e Lógica Prática: inferências múltiplas ${ }^{1}$
}

\author{
At the interface between Formal Logic and Practical Logic: multiple inferences
}

\author{
Jorge Campos da Costa² \\ Pontifícia Universidade Católica do Rio Grande do Sul - Porto Alegre - Rio Grande do Sul - Brasil
}

$\diamond$

\begin{abstract}
Resumo: $\mathrm{O}$ artigo que se segue representa a tentativa de demostrar que argumentos inválidos ditos falácias, ainda que assim o sejam num contexto formal, podem, num contexto natural, de argumentação prática, contar como coerentes relativamente a argumentos válidos. A questão principal seria a seguinte: esse tipo de situação ameaça a racionalidade natural ou cognitiva e comunicativa, à medida que argumentos válidos e inválidos são assumidos como corretos? A hipótese assumida é a de que não ocorre isso, e a explicação decorre de que há razões para se distinguir entre inferências multiformais, semânticas e pragmáticas para a abordagem do possível problema. Nesse caso, usaremos princípios de lógica clássica, de lógica modal e as regras deles decorrentes, especialmente a de Modus Ponens e a de Modus Tollens, e as falácias formais correspondentes, introduzindo as noções de interface, de inferências multiformes, relevância e condições de veracidade e comunicabilidade para a proposta de explicação, via uma suposição de racionalidade lato sensu. Reflexões de lógica informal e de raciocínio prático estão subjacentes ao longo de diversos argumentos aqui apresentados.
\end{abstract}

Palavras-chave: Lógica; Falácias; Relevância; Racionalidade; Semântica; Pragmática; Inferências multiformes

\begin{abstract}
Our paper is an attempt to demonstrate that fallacies, invalid arguments in formal logic, can be considered coherent and valid within a natural language context. What is at issue is if this kind of situation would threaten natural or cognitive and communicative reasoning, once both valid and invalid arguments are considered right. Our hypothesis is that this is not the case because there are ways and reasons to distinguish semantic and pragmatic inferences to deal with such a problem. We take specially the Modus Ponens and Modus Tollens rules, and the fallacy of the negation of the second premise and the converse error or affirming the consequent to reach an adequate description of the desirable explanation by using the notions of interface, multiple inferences, relevance and veracity conditions and communicative acceptability.
\end{abstract}

Keywords: Logic; Fallacies; Relevance; Reasoning; Semantics; Pragmatics; Multiform inferences

\section{Introdução}

Trata-se de aproximar a Lógica Dedutiva Clássica da Lógica da Linguagem Natural, na perspectiva de uma interface que pode trazer esclarecimentos sobre a natureza das relações entre propriedades inferenciais necessárias e contingentes. Numa interface formal, assume-se que $A \rightarrow B$ e $A$ levam dedutivamente de maneira necessária a $\mathrm{B}$, regra então chamada Modus Ponens. Em tal contexto,

\footnotetext{
1 Tradução do texto realizada por Ana Maria T. Ibaños e revisão de Yuri Penz.

2 Este artigo é uma versão aumentada e revisada do texto Multiform inferences: the interface between formal inferences and natural language inferences de Costa e Ibaños, publicado em Ekena Buja \& Stanca Mada (org.) Structure, Use and Meaning in Intercultural Settings. Brasov: Editura Universitä NII Transilvania
}

$A \rightarrow B$ e $\sim A$ não levam a $\sim B$, o que se chama falácia da negação do antecedente. De maneira análoga, $A \rightarrow B$ e $\sim B$ levam, dedutiva e necessariamente, a $\sim \mathrm{A}$, sendo a falácia da afirmação do consequente a que decorre de $A \rightarrow B$ e $B$, portanto, A. Numa interface natural com a linguagem natural, pode ser o que segue, pela ordem:

(1) Se João foi visto no local do crime com uma arma, então ele é o principal suspeito, ele foi visto no local do crime com uma arma; portanto, ele é o principal suspeito (válido).

(2) Se João foi visto no local do crime com uma arma, então ele é o principal suspeito, ele não foi visto no local do crime com uma arma; portanto, ele não é o principal suspeito (falácia). 
De modo similar,

(3) Se João foi visto no local do crime com uma arma, então ele é o principal suspeito, ele não é o principal suspeito; portanto, ele não foi visto no local do crime com uma arma (válido).

(4) Se João foi visto no local do crime com uma arma, então ele é o principal suspeito, ele é o principal suspeito; portanto, ele foi visto no local do crime com uma arma (falácia).

Dada, então, a pergunta sobre uma possível irracionalidade decorrente da aproximação de argumentos válidos e falácias, a hipótese que é assumida é a de que validade e invalidade, conceitos que se excluem logicamente, numa interface entre o formal, o natural ou cognitivo, e o comunicativo podem coexistir sem que se perca, radicalmente, a racionalidade. Para argumentar em favor da hipótese, seguem-se quatro seções: a primeira sobre a questão das interfaces formais, cognitivas e comunicativas; a segunda, já com os conceitos de aceitabilidade, condições de veracidade, interface semântica e pragmática, para a abordagem descritiva e explanatória do tópico; a terceira sobre a noção de inferências multiformais, e racionalidade lato sensu, e as considerações finais, ilustrações e exemplos que topicalizam os principais aspectos do presente texto.

\section{Interfaces entre o formal, o cognitivo e o comunicativo}

Uma abordagem teórica para esse tema, em que o que está em jogo são contextos diversos de racionalidade, implica alguns conceitos de aproximação interdisciplinar. Num primeiro momento, trata-se de assumir que a construção de interfaces quer dizer exatamente uma pavimentação argumentativa na intersecção interdisciplinar, mais propriamente multidisciplinar, entre Lógica, Linguística, Ciências Cognitivas e Comunicativas. Como se trata de uma questão sobre as condições de verdade e de validade, a Lógica está presente; a Linguística, com as condições de expressão e conteúdo semântico-pragmáticos da linguagem, as Ciências Cognitivas com as condições conceituais, ao nível do cérebro-mente, e as Ciências Comunicativas, com as condições dialógicas de aceitabilidade e veracidade. Obviamente, serão apenas chamadas a intervirem as noções próprias de cada área relativas ao problema em questão. Assume-se, primeiramente, uma distinção entre racionalidade stricto sensu e racionalidade em lato sensu. Uma peça argumentativa dedutiva é um objeto formal, lógico, como racionalidade do primeiro tipo; uma conversação argumentativa é um objeto linguísticocomunicativo, caracterizando racionalidade em sentido amplo.

Nas Ciências Cognitivas, é possível considerar os dois tipos de racionalidade, à medida que tem a ver com relações biossociais, como a noção de relevância, por exemplo. Em sentido restrito, o argumento está totalmente determinado pela forma lógica. Dado que $A$ é $B$ e $B$ é $C$, então $A$ é $C$; sendo que as metavariáveis e sua forma representam $n$ ocorrências de argumentos válidos. Pode-se chamar a isso de validade-tipo. Há, certamente, uma abstração do que se poderia denominar validadeocorrência, numa direção de perspectiva dependente de contexto. Então, levam-se em conta aspectos de validade-ocorrência dependentes de contextos diversos, especialmente o fato de que, com a linguagem natural, a semântica e a pragmática, participam, essencialmente, do processo de argumentar. De modo mais específico, paralelamente às condições formais da racionalidade em sentido restrito, emergem as formas do conteúdo que passam a constituir a estrutura de argumentação prática, ao lado das formas lógicas.

$\mathrm{Na}$ perspectiva formal, o contexto está reduzido aos esquemas de regras; na comunicativa, aos contextos variados. Por trás, evidentemente, está a diferença entre abstração e uso de argumentos, ou mais tradicionalmente, de aplicação de argumentos. No nível formal, levam-se sempre em conta as condições-de-verdade; nos diálogos argumentativos em linguagem natural, talvez seja mais adequado falar-se em condições de veracidade ou de aceitabilidade da verdade em seu contexto.

Considere o seguinte argumento, que poderia ser parte de um diálogo:

(5) Todos os políticos são corruptos e se João é político, então João é corrupto.

se a noção dura de verdade fosse invocada, ficaria comunicativamente congelada, dada a impossibilidade de verificação da primeira premissa. Mas, se $A$ argumenta com $B$ em defesa do argumento em questão, $B$ pode aceitá-lo como veraz, ou razoável, claro, num contexto de comunicação trivial, não científico. De um ponto de vista cognitivo, por exemplo, as condições de aceitabilidade comunicativa poderiam ser determinadas por condições de conceitos e de crenças. A cognição humana, na perspectiva da Teoria da Relevância (SPERBER e WILSON, 1986, 1995, 2005) é caracterizado por dois princípios fundamentais da relevância: $\mathrm{O}$ da cognição e o da comunicação. $\mathrm{O}$ primeiro consiste em assumir-se que há uma tendência inata do cérebro/mente humano para a relevância, e o segundo que, comunicativamente, tenta-se ser o mais 
relevante possível. A relevância, nesse contexto, é uma relação otimizada entre custo-benefício, cognitivamente, é claro. Quanto menor for o custo e maior o benefício, maior será a relevância (WILSON e SPERBER, 1981, 1986). Assim, a noção essencial de inferência numa perspectiva interdisciplinar assume diversas formas. Numa interface formal, ela tem a formatação dedutiva. É uma inferência necessária e normativa. Numa interface cognitivo-comunicativa, a inferência é a posteriori e não normativa. Ela surge de uma conexão entre um contexto e uma proposição nova. No caso, a inferência contextual não é tópica e tautológica como na Lógica, mas é nãotrivial, complexa e global. A próxima seção discute as possíveis abordagens desses tipos de inferências, endossando as palavras de Toulmin que justificam o nosso trabalho: "Habits of inference [...] begin by being merely customary, but in due course become mandatory or obligatory" (2003, p. 4).

\section{Diálogos argumentativos, falácias, validade e relevância}

Voltemos ao problema formulado no início, descrevendo a questão problemática da aproximação entre falácias formais e argumentos dedutivos válidos. A dificuldade primeira é o porquê de as falácias formais serem argumentos inválidos especiais no conjunto das invalidades; o segundo problema está no fato de que, ainda inválidas, as tais falácias são aceitas ao nível do senso comum, e o terceiro é quais razões podem ser levantadas para justificar algum tipo de racionalidade nos casos de Modus Ponens e Modus Tollens. Inicialmente, as falácias formais são assim identificadas porque elas representam regras praticamente simétricas às válidas. A afirmação do antecedente do condicional, leva ao seu consequente, e a negação de seu consequente leva à negação de seu antecedente é, estruturalmente, apenas uma troca de ordem para a negação de seu antecedente leva à negação de seu consequente e a afirmação de seu consequente leva à afirmação de seu antecedente. Assim, a semelhança poderia ser uma razão para as falácias formais. Elas são formalmente semelhantes às válidas, o que significa uma diferença para as invalidades não formais. Poder-se-ia considerar que "semelhança" não é uma propriedade lógica considerável, mas semelhança formal tem outra dimensão; a segunda situação envolve a primeira. A semelhança de formas lógicas faz com que o pacote das quatro situações apareça como mais razoável do que duas validades e duas invalidades. Tal raciocínio pode parecer, à primeira vista, externo à verdadeira diferença ao nível lógico, mas veja-se que, na perspectiva em jogo, tudo é formal. Se for levado o conteúdo a esclarecer qualquer aspecto, eis que se passa a interferir na forma lógica, através da forma do conteúdo. Mas isso já não é Lógica stricto sensu.

Considere-se, então, o caso, via argumento prático, ou cognitivo-comunicativo, com a noção de relevância e a forma do conteúdo em questão, na ilustração dialógicoargumentativa de um dono de carro e um lavador de rua.

(6) (A) Se lavas meu carro, dou-te 10 reais.

(B) Não consegui lavar todo.

(A) Então não vou te pagar.

(B) Por que não? Você não falou da hipótese de eu não lavar todo.

O argumento de (A) é o de uma falácia, a saber, a negação do antecedente para a negação do consequente. De fato, o proprietário do carro, ao contratar o serviço, gerou uma inferência pragmática de bicondicional (te dou dez reais se e somente se lavares todo o carro). Essa inferência contextual validaria o argumento, mas o lavador a cancela, como pode ocorrer com inferências pragmáticas, ao dizer que o dono do carro não havia mencionado a hipótese de ele não lavar todo. Ou seja, ambos têm e não tem razão. Contradição? Não exatamente. Ao fazer a proposta para o lavador, se houvesse a hipótese de não se inferir o bicondicional de maneira compartilhada, a proposta inicial seria irrelevante. Algo como se ou não lavares todo o carro, terás dez reais. Cognitivamente, o argumento sem a determinada inferência, traria menos benefícios do que com a inferência, pelo menos se se assume a hipótese de que a decodificação tem custo maior no caso da comparação dito/implícito. Ou seja, o argumento se lavares meu carro e só se o lavares todo, terás os 10 reais teria mais custo operacional, com a explicitação do bicondicional, do que gerando uma inferência pragmática. E por que a inferência seria normalmente aceita por ambos? Porque ela seria a mais relevante dado o mesmo custo inicial com o bicondicional inferido. Ou seja, condicionais são frequentemente interpretados como bicondicionais porque tal interpretação é mais relevante. O condicional teria as duas partes explícitas $\mathrm{P}$ e $\mathrm{Q}$ de custo e duas informações de benefício, mais o benefício de inferência de bicondicional. Caso contrário, na explicitação do bicondicional, haveria o $\mathrm{P}$, o Q e mais o bicondicional de custo, para os mesmos três benefícios. Poder-se ia, sem dúvida, considerar que a explicitação do bicondicional seria um benefício mais forte, o que deixaria o jogo comparativo empatado. Mas parece que o fato no senso comum é pela não explicitação do bicondicional. As pessoas mais raramente o usam. Bem, isso poderia ser um indício de que entre a inferência e o dito explícito, nosso cérebro/mente opta pela inferência. $\mathrm{Na}$ verdade, o bicondicional é constituído de dois condicionais então, se ele estiver implícito, isto pode ser uma regra de pragmática 
inferencial, um caso default de condicional para inferência do bicondicional. Caso isso seja verdadeiro, o uso do default também é razão de economia, o que é consistente com a noção de relevância. Isso endossa, também, a vantagem de argumentos práticos no uso de inferências necessárias e canceláveis ao mesmo tempo, aumentando a relevância da porção de racionalidade envolvida. Talvez, e o diálogo pode ser um exemplo, tudo esteja no equilíbrio entre o inferir e o dizer, de modo que o dito é mais caro e tem menos risco; enquanto o inferível é mais barato e também mais arriscado. Mas como Récanati (2002, p.3) salienta,

There is no limit to the amount of contextual information that can in principle affect pragmatic interpretation. Any piece of information can turn out to be relevant and influence the outcome of pragmatic interpretation.

A Seção 4 discute as possibilidades de se considerar inferências em multitude formas.

\section{Sobre inferências multiformais}

Nesta secção, estaremos tratando de inferências que podem ser construídas em múltiplas interfaces. Isso quer dizer que o avanço das teorias sobre o binônimo significado da sentença/significado do falante, no roteiro original griceano, vem abrindo perspectivas para o desenvolvimento de uma Pragmática Inferencial em oposição ao modelo clássico de códigos (SHANNON e Weaver, 1949), como argumentam Sperber e Wilson (1983). Numa generalização maior, o que parece, quando aplicamos uma versão de Navalha de Ockam, é que a noção de inferência pode ser entendida enquanto múltiplas formas de raciocinar a partir do dito e de infinitos contextos. Nesse sentido, é que se pode vislumbrar uma possibilidade de relações de interface externa, ou interdisciplinares, e interfaces internas ou intradisciplinares, envolvendo a aproximação de áreas e subáreas respectivamente.

Como estamos ao nível do que se poderia chamar de ciências da linguagem, a relação da Linguística com a Lógica Clássica caracteriza uma perspectiva interdisciplinar entre as duas grandes áreas, sendo que as interações entre subáreas, como a lógica proposicional e a semântica ou pragmática, representam articulações internas ou intradisciplinares. Assim, cabe explorar os processos inferenciais ao nível das diversas interfaces. Tomemos a Linguística Teórica e suas subáreas Fonética (Fonologia), Morfologia, Lexicologia, Sintaxe, Semântica e Pragmática, em suas relações de interface intradisciplinar, do som ao sentido para simplificar.
Podemos examinar inferências ao nível da prosódia, lexical, semântica, etc. Considere os exemplos que se seguem:

(7) Quem é você? QUEM é você? Quem é VOCÊ?

(8) Quem é você!

(9) João vai reiniciar sua dieta.

(10) Amanda ganhou algumas rosas em seu aniversário.

(11) Amanda ganhou flores em seu aniversário.

(12) João vendeu uma casa para Maria.

(13) Uma casa foi vendida para a Maria por João.

(14) Todos são culpados.

(15) Alguns são culpados.

(16) Apenas poucos ficaram na palestra de João até o final.

(17) Muitas pessoas não gostaram da fala de João.

Para ilustrar, uma pergunta como "quem é você?", implica um processo inferencial disparado pela prosódia para pergunta e resposta; já "Quem é você!", como prosódia de exclamação, pode detonar uma inferência de surpresa entendida pelo interlocutor, como algo do tipo "quem é você para ousar fazer isso." Ao nível da Morfologia, os prefixos, sufixos, entre outros tipos de morfemas, também disparam processos inferenciais. Quando alguém diz "vou retomar a minha dieta" leva seu interlocutor a depreender que havia uma dieta anterior que tinha sido abandonada. Isso em decorrência do prefixo "re", que gera inferências de pressuposição. De um ponto de vista lexical, "rosa" e "flor" mantêm uma relação de acarretamento, um tipo de inferência em que "isto é uma rosa" acarreta que "isto é uma flor". Toda vez que a primeira for verdadeira, a segunda também o será. De maneira similar, uma inferência sinonímica pode ser obtida de duplo acarretamento, quando $A$ acarreta $B$ e $B$ acarreta $A$. Na mesma direção, agora sintática, de "João vendeu uma casa para Maria" infere-se que "uma casa foi vendida por João para Maria", caso de passiva. Também ao nível de uma proposição, ou seja de objeto semântico, de "todos são culpados" há uma inferência para "alguns são culpados". Ao mesmo tempo, no âmbito pragmático, de "poucos assistiram à palestra até ao final", pode-se inferir que "muitos não gostaram da palestra".

Da mesma forma, num contexto de unidades dialógicas, uma pergunta leva a inferência de que há uma expectativa de resposta. Se alguém me pergunta se eu sei que horas são, devo inferir que, estando informado, devo responder dizendo as horas. Nesse ponto, então, podem ser identificados gatilhos inferenciais desenhados nas diversas subáreas da Linguística. Conforme diversos modelos de abordagem, via Grice $(1957,1989)$, Gazdar (1979), Sperber e Wilson (1986), Levinson, (2000), entre 
outros, são identificadas inferências de vários tipos, implicaturas, pressuposições, acarretamentos, inferências lógico-dedutivas, implicações contextuais, inferências conversacionais, dialógicas, inferências monotônicas, não-monotônicas, etc.

$\mathrm{Na}$ verdade, até o próprio conteúdo do que é dito é determinado por processos inferenciais. Isto significa que, conforme o anteriormente mencionado, assume-se que a racionalidade stricto sensu está incluída na racionalidade lato sensu, cuja amplitude é nutrida pelo jogo de operações inferenciais complexas, ou de propriedades multiformais. Nesse sentido, a inferência dedutiva natural faz parte do referido conjunto à medida que raciocinamos naturalmente com o condicional e afirmação do antecedente para concluir o consequente.

Suponhamos um argumento cotidiano,

(18) Se os candidatos ao governo são corruptos, então não votarei, há evidências positivas para o caso; não votarei, portanto.

Na interface lógico-linguística, $P \rightarrow Q$ and $P-\mid Q$ é a forma lógica-tipo, o modus ponens, subjacente à ocorrência do argumento em pauta com o conteúdo adequado. Trata-se de um raciocínio prático, envolvendo um processo dedutivo natural (IBAÑOS, 2005). Na mesma rota, os princípios fundamentais na tradição aristotélica, o da não-contradição, $\sim(A \& \sim A)$, o de identidade, $A=A$, e o do terceiro excluído, $A V \sim A$, sustentam o edifício formal da Lógica Clássica, mas não deixam de fazer sentido numa perspectiva de raciocínio prático.

O primeiro diz que não é o caso que uma proposição e sua negação sejam verdadeiras ao mesmo tempo sob a mesma circunstância. Isso na dimensão da Lógica stricto sensu. Na concepção de uma racionalidade prática mais ampla significa que a contradição, a incoerência, a inconsistência são propriedades que ameaçam o raciocínio válido, a aceitabilidade da derivação, mas sem impedir que tal princípio seja desafiado.

Dada uma diferença de fases na vida de Maria, a adolescência e a adultez, não é irracional que se diga que ela é virgem e não é virgem respectivamente, que ela faz sexo e não faz sexo, conforme as circunstâncias. De maneira análoga, a noção na Lógica de que de uma contradição se siga qualquer coisa corresponde, no raciocínio prático, aos efeitos sobre os usos de argumentos com suposições inconsistentes, incoerentes, das quais se diz que contaminam a aceitabilidade do processo argumentativo. Ainda assim, conforme se considerou anteriormente, na comunicação dialógica $A$ diz para $B$ que ele, $B$, está sendo incoerente não tem a devastadora consequência de que tudo se siga dali por diante. $A$ aceita que a inconsistência de $B$ possa ser local, e a argumentação continue. Nas palavras de Mercier e Sperber (2011, p. 60)

the main function of reasoning is argumentative: Reasoning has evolved and persisted mainly because it makes human communication more effective and advantageous.

Quanto ao segundo princípio, o que ele diz é que, dada uma proposição verdadeira $A$, ela é idêntica a si mesma, ou seja, ela mantém a sua identidade, ou, ainda, ela não pode não ser ela mesma. Intuitivamente, numa perspectiva comunicativo-dialógica, a lei da identidade corresponde a que uma coisa identificada como $A$ será sempre assim identificada em qualquer contexto possível. Não fosse assim, se a perda ou mudança da identidade fosse assumida, a verdade poderia ser deslocada a cada momento e a racionalidade perdida. Mas, mesmo dessa forma, nada impede que $A$ diga para $B$ "naquele momento eu não era eu" para referir-se a alguma perda de propriedade essencial, obviamente via metáfora.

A terceira e última lei, a do terceiro excluído, significa que uma proposição é sempre verdadeira ou falsa, sem alternativa. Isso, efetivamente, é a defesa de uma lógica bivalente que protege o desenvolvimento de uma racionalidade científica. Mas, do ponto de vista comunicativo-dialógico, há uma tradição da bivalência sem que isso afete a ideia clara de que pode haver um meio termo como, por exemplo, morno entre frio e quente, ou altura média entre alta e baixa, etc. Nada impede, rigorosamente, que haja perspectivas trivalentes ou, mesmo, polivalentes, e que uma racionalidade se mantenha nessa direção. Um ideia de três valores, como o verdadeiro, o falso e o nem verdadeiro nem falso, pode vir a ser uma solução para as falhas de pressuposição existencial como é o famoso caso de "o Rei da França é calvo", quando o Rei da França não existe, situação em que a proposição e sua negação são ambas falsas. Além dos operadores proposicionais já mencionados, os modais e quantificacionais expressam, também, conflitos nas interfaces com conflitos inferenciais. Encerramos a seção, então, com uma avaliação de interface ao nível de argumentos dedutivos e argumentos práticos, quanto às modalidades e quantificações. Também ao nível das modalidades, como a necessidade e a possibilidade, as relações em termos de lógica Modal e de uso da lógica natural, para fins cotidianos, expressam aspectos conflitantes e problemáticos. Suponha-se a sequência argumentativa, primeiramente em sua forma lógica em que estão em jogo as noções de necessidade e possibilidade de é necessário que $\mathrm{P}$ e que é possível que $\mathrm{P}$. 
(19)

$$
\begin{aligned}
& 1 \square \mathrm{P} \rightarrow \diamond \mathrm{P} \\
& 2 \diamond \mathrm{P} \rightarrow \diamond \sim \mathrm{P} \\
& 3 \triangleleft \sim \mathrm{P} \rightarrow \sim \square \mathrm{P} \\
& 4 \sim \square \mathrm{P} \rightarrow \square \mathrm{P}
\end{aligned}
$$

1 É necessário que $\mathrm{P}$ implica que é possível que $\mathrm{P}$

2 É possível que $\mathrm{P}$ implica que é possível que não $\mathrm{P}$

3 É possível que não $\mathrm{P}$ implica que não é necessário que $P$

4 Não é necessário que P implica que é necessário que $\mathrm{P}$

À primeira vista, o argumento é válido, a sequência se segue naturalmente, e a dedutibilidade se propaga numa racionalidade consensual. A questão, entretanto, que expressa a contradição está na última linha do argumento. Como pode ser que seja necessário que $\mathrm{P}$ implique que não seja necessário que P. Ocorre que a linha dois é inválida à medida em que ela leva a uma conjunção contraditória $N P \& \sim N P$. Ou seja, as três primeiras linhas parecem autorizar a 4, mas esta é uma proposição inconsistente. Por que isto é assim? porque é possível que $\mathrm{P}$ não leva à necessária dedução de que é possível que não P. Mas, intuitivamente, se algo é possível, algo não é possível.

Veja-se, ao nível dos quantificadores "Qualquer que seja x" e "Existe pelo menos um x". Analogamente:

$$
\begin{aligned}
& 1((\forall \mathrm{x}(\mathrm{Px} \rightarrow \mathrm{Cx}) \rightarrow((\exists \mathrm{x})(\mathrm{Px} \& \mathrm{Cx})) \\
& 2((\exists \mathrm{x})(\mathrm{Px} \& \mathrm{Cx})) \rightarrow((\exists \mathrm{x})(\mathrm{Px} \& \sim \mathrm{Cx}) \\
& 3((\exists \mathrm{x})(\mathrm{Px} \& \sim \mathrm{Cx}) \rightarrow \sim(\forall \mathrm{x})((\mathrm{Px} \rightarrow \mathrm{Cx}) \\
& 4 \sim(\forall \mathrm{x})((\mathrm{Px} \rightarrow \mathrm{Cx}) \rightarrow \forall \mathrm{x}((\mathrm{Px} \rightarrow \mathrm{Cx}) \\
& 1 \text { Todos os políticos são corruptos implica que } \\
& \text { alguns o são } \\
& 2 \text { Alguns políticos são corruptos implica que alguns } \\
& \text { políticos não são corruptos } \\
& 3 \text { Alguns políticos não são corruptos implica que nem } \\
& \text { todos políticos são corruptos } \\
& 4 \text { Nem todos os políticos são corruptos implica que } \\
& \text { todos políticos são corruptos }
\end{aligned}
$$

Novamente, a última linha é contraditória, porque a segunda não é legítima, ainda que seja intuitivamente como tal. As questões interessantes, então, são as que se expressam na interface entre a Lógica stricto sensu e о pensamento crítico, ou Lógica Informal, ainda entendida como uso de argumentos. Por que o uso dos argumentos é intuitivamente válido em situações dessas? A segunda linha em ambos os casos parece ser tratável de um ponto de vista comunicativo-cognitivo. Vamos à Teoria de Relevância (TR) de Sperber e Wilson. Nela assumem-se dois princípios em que o primeiro defende uma tese de que há uma tendência da cognição humana para a relevância, e um segundo, em que há uma tendência para uma maximização da relevância enquanto custo e benefício.
No caso da linha 2, em ambos os casos, a inferência pragmática de que se é possível que sim, é possível que não; e a de que, se alguns que sim, então alguns que não, parece razoável. De fato, nos diálogos abaixo:

(21) 1 João: Não sei se viajaremos, é possível que chova 2 Maria: mas, se não chover, iremos sem dúvida 3 Com certeza

Maria inferiu, pragmaticamente, de "é possível que chova" "é possível que não chova" em cuja hipótese, João confirma a viagem, aceitando, portanto, a inferência de Maria,

(22) 1 João: Alguns amigos irão conosco

2 Maria: mas alguns perderão a oportunidade

3 João: o problema é deles

Maria inferiu de "alguns amigos irão conosco" que "alguns não irão" para concluir que, os que não forem, irão perder a oportunidade.

A inferência pragmática, em ambos os casos, pode ser atribuída a uma maior relevância, tendo em vista que o enunciado "é possível que chova" logicamente leva à inferência "não é necessário que não chova", mas será mais relevante se dele for inferido adicionalmente que é possível que não chova. Da mesma forma, de "alguns irão conosco" Maria infere "alguns não irão conosco" o que representa uma informação adicional pragmaticamente mais relevante.

\section{Considerações finais, exemplos e ilustrações}

Desde que Aristóteles distinguiu argumentos apodíticos de dialéticos, um mundo de investigações se abriu na interface entre Lógica e Linguagem Natural. A questão sobre a validade de um argumento pode ser projetada livre de contexto no nível da forma lógica ou dentro de um contexto de debates dialógicos (EEMEREN e GROOTENSDORST, 1992; PINTO, 2001, 2006). O primeiro representa uma perspectiva de um estudo teórico, enquanto que o segundo representa características comunicativas práticas. É possível e desejável investigar essas duas dimensões de raciocínio via linguagem natural, quando existe um conflito na interface lógica-linguagem.

As considerações que se seguem topicalizam o mais relevante.

(a) Assuma-se a distinção entre racionalidade estrita e racionalidade ampla;

Trata-se de considerar a interface entre uma área formal, a Lógica Clássica, uma área comunicativo- 
social, a Linguística, e uma área cognitiva, a Teoria da Relevância como ponte interdisciplinar. A primeira delas caracteriza-se pelo que assumimos como racionalidade em sentido restrito, livre de contexto, de caráter descritivo-normativo, em que o processo inferencial é algorítmico e trivial; a segunda é o que assumimos como racionalidade em sentido amplo, dependente de contexto e de fatores comunicativos dialógicos, em que os processos inferenciais são múltiplos, ou multiformes; a terceira é a área cognitiva base para ambas as formas de racionalidade acima mencionadas;

(b) Assuma-se a distinção argumentos dedutivos e argumentos naturais, sendo que os primeiros podem estar no conjunto dos segundos;

$\mathrm{Na}$ esteira do que se propõe como raciocínio formal, matemático, e raciocínio comunicativosocial, trata-se de separar os dois tipos de argumento, o esquemático abstrato e o dialógico interativo. $\mathrm{O}$ primeiro vai na direção matemático-computacional, e o segundo na direção do persuasivo e intencional. Também é possível distinguir, em bases semelhantes, a inferência monotônica e a não-monotônica;

(c) Assumindo-se o que se diz nos dois itens anteriores, no conjunto mais amplo das inferências multiformais, encontram-se as inferências restritas, dominadas por regras e de caráter trivial e as globais, nãodemonstrativas e não-triviais. Isso significa que os argumentos lógico-dedutivos são apenas um subconjunto dos argumentos práticos, que resultam de operações lógico-cognitivo-comunicativas. Isto significa dizer que a racionalidade ampla é complexa e deve ser abordada interdisciplinarmente ou via construção de interfaces;

(d) $\mathrm{Na}$ perspectiva de tal interface, aproximam-se as noções de condições-de-verdade para os argumentos lógico-formais e condições-de-veracidade ou de aceitabilidade para argumentos dialógicos ou práticos. Estes últimos incluem os primeiros, aqueles que são dependentes de contexto teórico ou abstrato, ou livres de contextos comunicativos específicos; os primeiros estão relacionados às formas lógicas válidas ou inválidas, enquanto os segundos às formas do conteúdo de que se abstraem os primeiros;

(e) A noção de validade ou de invalidade está para o raciocínio lógico, assim como a noção de relevância está para o raciocínio cognitivo-comunicativo. A distinção e aproximação entre argumentos apodíticos e dialéticos da tradição aristotélica apontam para interfaces cognitivas em que cérebro-mente parecem ter propriedades subjacentes a raciocínios formais e multiformais da racionalidade ampla; (f) Quanto à distinção entre Semântica e Pragmática, ambas podem ser dependentes de interface. Ambas podem ser formais, cognitivas ou comunicativas, e ambas podem ser aplicadas às duas perspectivas de racionalidade. No sentido de uma interface comunicativo-social, destaca-se o caráter de uso da linguagem ao nível de enunciados e porções discursivas, dependentes de contexto, ficando a Semântica típica com a descrição de proposições livres de contexto. Há uma tensão metodológica entre as duas subáreas da Linguística, com direções da Pragmática para abranger os fenômenos ditos semânticos, cuja indeterminação parece ser um indício que aponta para o avanço dos modelos de uso;

(g) Quanto às interfaces com a Lógica Clássica e Complementação Modal, entendem-se os quantificadores e modalidades como presentes na argumentação formal e na argumentação prática, pressupondo racionalidade na ordem do plausível, ou razoável;

(h) Ilustração de abordagem interdisciplinar Lógica Clássica/Linguística Comunicativo-social/ Linguística Cognitivo-social;

- perspectiva interdisciplinar e de construção de interfaces:

Exemplo o "\&" objeto lógico, o "e" objeto linguístico-comunicativo e o "e/\&", objeto cognitivo. O " \&" tem a propriedade comutativa P\&Q $<>Q \& P$ "João é advogado e Maria é médica" e "Maria é médica e João é advogado" - mesmas inferências

$\mathrm{O}$ "e" não tem a propriedade comutativa "João pegou o dinheiro e foi ao banco" e "João foi ao banco e pegou o dinheiro" - inferências diferentes

$\mathrm{O}$ "e/\&" não tem a propriedade comutativa - "Carlos acredita que João foi dirigindo para casa e bebeu um litro de uísque" mas "Carlos não acredita que João bebeu um litro de uísque e foi dirigindo para casa"

João A (P e/\& Q) e A (Q e/\& P) ou seja, dada uma ordem no tempo, o raciocínio prático encontra uma inferência do tipo $\mathrm{P}$ e, então, $\mathrm{Q}$ ou $\mathrm{Q}$ e, então, $\mathrm{P}, \mathrm{o}$ que evita que haja um contradição.

Dado um diálogo argumentativo como o abaixo:

A Se João tomou um litro de uísque e (então) foi dirigindo para casa, ele será preso. ( $\mathrm{P}$ e/\& Q)-> R

$B$ Mas se foi dirigindo para casa e (então) bebeu um litro de uísque, será preso por isso? (Q \&/e P)->R?

Isso é assim porque o "e/\&" envolve uma inferência de sequência de ações gramaticalizada como "e então". A razão de que, ainda que não necessariamente, apareça com frequência uma inferência associada ao "e" pode ter uma explicação por relevância. Dada 
a expressão molecular"P e/\& Q,", as condições de verdade são as de que P seja verdadeira e Q também o seja, independentemente da ordem. $\mathrm{Na}$ interface comunicativo-social, além das duas proposições, as condições de veracidade implicam uma inferência de ordem como uma terceira informação, o que representa uma implicação cognitiva a enriquecer, tornar mais relevante, a proposição molecular com uma inferência, do que a mesma sem ela. De fato, para o argumento prático, a conjunção parece levar a expectativas de inferência quanto à forma de conexão aditiva. A primeira proposição parece expressar relevância à precedência. É o caso da causa. "João vinha correndo e caiu" parece ser mais relevante do que "João caiu e vinha correndo", quando a segunda parece com maior custo que a primeira, já que o natural é que a precedência semântica da causa seja mais natural com a precedência sintática da ordem. Com polissíndetos, fica mais claro: "João vinha correndo e tropeçou numa pedra e desequilibrou-se e caiu" apresenta-se com menor custo, mais relevante, portanto, se comparada a "João caiu e desequilibrouse e tropeçou e vinha correndo".

Uma certa dificuldade é natural nas interfaces interdisciplinares lógico-cognitivo-comunicativas. O desafio é articulá-las. O prêmio uma certa intuição de que é um roteiro interessante na direção da complexidade.

\section{Referências}

ARISTOTLE. The works of Aristotle. Chicago: William Benton, 1953.

CAMPOS, Jorge. The sciences of language: communication, cognition and computation - Inter/Intradisciplinary Relations. In: AUDY, J.L.N.; MOROSINI, M.C. (Orgs.). Innovation and interdisciplinarity in the University / Inovacão e interdisciplinaridade na Universidade. Porto Alegre: EDIPUCRS, 2008. p. 345-360.

COSTA, Jorge Campos. Lógica e Linguagem Natural nas interfaces. In: COSTA, J. C.; PEREIRA, V. W. Linguagem e cognição: relações interdisciplinares. Porto Alegre: EDIPUCRS, 2009. p. 132-142.

GAZDAR, G. Pragmatics: implicature, presupposition, and logical form. New York: Academic Press, 1979.
GRICE, H.P. Meaning. The philosophical review, p. 377-388, 1957.

GRICE, H.P. Studies in the Way of Words. Cambridge, MA: Harvard University Press, 1989.

IBAÑOS, Ana Maria Tramunt. Algumas considerações informais sobre inferência. In: Linguagem em (Dis)Curso, v. 5, p. 151-159, 2005.

LEVINSON, Stephen. Presumptive Meanings. Cambridge, MA: MIT, 2000.

MERCIER, H.; SPERBER, D. Why do humans reason? Arguments for an argumentative theory. In: Behavioral and Brain Sciences, v. 34, n. 2, p. 57-74, 2011.

PINTO, R.C. Argument, Inference and Dialectic. The Netherlands: Kluwer Academic Publishers, 2001.

PINTO, R.C. Evaluating Inferences: the nature and role of warrants. In: Informal Logic, v. 26, n. 3, p. 287-317, 2006.

RÉCANATI, François. Does Linguistic Communication Rest on Inference? Mind and Language, v. 17, n. 1-2, p. 105-126, 2002.

SHANNON, C.E.; WEAVER, W. The mathematical theory of communication. Urbana, Illinois: University of Illinois Press, 1949.

SPERBER, Dan; WILSON, Deirdre. 1986. Relevance: Communication and Cognition. 2. ed. Oxford: Blackwell, 1995.

SPERBER, Dan; WILSON, Deirdre. Teoria da Relevância. Linguagem em (Dis)curso, Tubarão, v. 5, p. 221-269, 2005.

TOULMIN, S. E. 1958. The uses of argument. Cambridge: Cambridge University Press, 2003 (updated edition).

VAN EEMEREN, F. H.; GROOTENDORST, R. Argumentation, Communication, and Fallacies: a pragma-dialectical perspective. Hillsdale, NJ: Lawrence Erblaum Associates, 1992.

WALTON, D. Argument Structure: a pragmatic theory. Toronto: University of Toronto Press, 1996.

WILSON, D.; SPERBER, D. On Grice's theory of conversation. In: WERTH (Ed.). Conversation and discourse. London: Croom Helm, 1981. p. 155-78. [Reprinted in KASHER (Ed.), 1998. Vol. IV, p. 347-368].

WILSON, D.; SPERBER, D. Pragmatics and modularity. Chicago Linguistic Society 22, parasession on pragmatics and grammatical theory. 1986. p. 68-74.

Recebido: 03 de fevereiro de 2016.

Aprovado: 02 de junho de 2016.

Contato: jcampos@pucrs.br 